\title{
Anosmia and dysgeusia associated with SARS-CoV-2 infection: an age-matched case-control study
}

\author{
Alex Carignan MD MSc, Louis Valiquette MD MSc, Cynthia Grenier MSc, Jean Berchmans Musonera MD, \\ Delphin Nkengurutse MD, Anaïs Marcil-Héguy MSc, Kim Vettese MSc, Dominique Marcoux RN, \\ Corinne Valiquette, Wei Ting Xiong, Pierre-Hughes Fortier MD, Mélissa Généreux MD MSc, \\ Jacques Pépin MD MSc
}

Cite as: CMAJ 2020 June 29;192:E702-7. doi: 10.1503/cmaj.200869; early-released May 27, 2020

\begin{abstract}
BACKGROUND: Anosmia and dysgeusia have been reported as potential symptoms of coronavirus disease 2019. This study aimed to confirm whether anosmia and dysgeusia are specific symptoms among those who tested positive for severe acute respiratory syndrome coronavirus 2 (SARS-CoV-2).
\end{abstract}

METHODS: We conducted an age-matched case-control study in the Eastern Townships region of Quebec between Mar. 10 and Mar. 23, 2020. We included adults (age $\geq 18 \mathrm{yr}$ ) who tested positive for SARS-CoV-2 by reverse transcription polymerase chain reaction. Cases were matched (1:1) according to 5-year age groups with con- trol patents selected randomly from among all patients who tested negative for SARS-CoV-2 during the same period. Demographic and laboratory information was collected from medical records. Clinical symptoms and comorbidities associated with anosmia and dysgeusia were obtained by telephone interview with a standardized questionnaire.

RESULTS: Among 2883 people tested for SARS-CoV-2, we identified 134 positive cases (70 women [52.2\%] and 64 men [47.8\%]; median age 57.1 [interquartile range 41.2-64.5] yr). The symptoms independently associated with SARS-CoV-2 positivity in conditional logistic regres- sion were anosmia or dysgeusia or both (adjusted odds ratio [OR] 62.9, 95\% confidence interval $[\mathrm{Cl}]$ 11.0-359.7), presence of myalgia (adjusted OR 7.6, 95\% Cl 1.929.9), blurred vision (adjusted OR 0.1, 95\% $\mathrm{Cl} 0.0-0.8$ ) and chest pain (adjusted OR 0.1, 95\% Cl 0.0-0.6).

INTERPRETATION: We found a strong association between olfactory and gustatory symptoms and SARS-CoV-2 positivity. These symptoms should be considered as common and distinctive features of SARS-CoV-2 infection and should serve as an indication for testing and possible retesting of people whose first test result is negative. n

n the current coronavirus disease 2019 (COVID-19) pandemic, anosmia and dysgeusia have been described as potential symptoms of the disease. On Mar. 21, 2020, a press release from ENT UK (a professional membership body representing ear, nose and throat surgeons in the United Kingdom) and the British Rhinological Society reported anosmia as a symptom in up to $40 \%$ of patients in China, South Korea, Germany and Italy. ${ }^{1}$ Surprisingly, anosmia and dysgeusia were not reported in the first study describing the clinical characteristics of COVID-19 in China. ${ }^{2}$

Severe acute respiratory syndrome coronavirus 2 (SARSCoV-2) damages primarily the respiratory tract. The most common symptoms of SARS-CoV-2 infection include fever, cough and muscle pain. ${ }^{3,4}$ Although most patients generally experience mild to moderate disease, severe or critical disease requiring hospital admission develops in $15 \%-20 \%$ of patients, with an overall fatality rate of $2.3 \% .^{5}$

A growing body of literature has mentioned anosmia and dysgeusia as potential symptoms of SARS-CoV-2 infection. ${ }^{1,6,7}$ Anosmia is associated with other respiratory tract infections, and it is not clear whether this symptom is a consequence of nasal obstruction and congestion, or is a symptom specific to SARSCoV-2 infection. ${ }^{8}$ A better understanding of the association between these symptoms and SARS-CoV-2 infection might update the diagnostic criteria and alert people who experience these symptoms to isolate early and seek testing. Therefore, we aimed to delineate the value of anosmia and dysgeusia as potential specific symptoms of SARS-CoV-2 infection. 


\section{Methods}

\section{Population and design}

This was an age-matched case-control study conducted in the Eastern Townships region of Quebec between Mar. 10 and Mar. 23, 2020. The Centre intégré universitaire de santé et de services sociaux de l'Estrie-Centre hospitalier universitaire de Sherbrooke (CHUS) provides hospital care to the 170000 residents of Sherbrooke as well as referral services for the Estrie region (total population 508000) in southern Quebec. The study population included all patients who underwent testing for SARS-CoV-2 during this period at the CHUS, whose laboratory is responsible for all SARS-CoV-2 testing in the region.

\section{Selection of case and control participants}

Data were extracted from the CHUS clinical data warehouse to identify all adult (age $\geq 18 \mathrm{yr}$ ) patients who underwent testing for SARS-CoV-2 by means of in-house reverse transcription polymerase chain reaction (RT-PCR) between Mar. 10 and Mar. 23, 2020. The assay limit of detection is 200 SARS-CoV-2 RNA copies/ $\mathrm{mL}$. During this early period, there was almost no known community transmission in the region, and the criteria for testing were a temperature greater than $38^{\circ} \mathrm{C}$ or newly occurring cough or dyspnea, and epidemiologic criteria (travel to a country with known COVID-19 cases in the previous $14 \mathrm{~d}$, or contact with a confirmed COVID-19 case or with a person with acute respiratory disease who had travelled to a region with known COVID-19 cases, or laboratory exposure to biologic material known to contain SARS-CoV-2). For each case, control patients were matched (1:1) according to 5-year age groups selected by means of a pseudorandom number generator from all patients who tested negative for SARS-CoV-2 at the CHUS during the same period. Patients with multiple tests during the study period were excluded.

\section{Standardized questionnaire}

All participants were interviewed via telephone by trained interviewers using a standardized questionnaire (Appendix 1, available at www.cmaj.ca/lookup/suppl/doi:10.1503/cmaj.200869/-/ DC1). We adapted questions from the self-reported Mini Olfactory Questionnaire (Self-MOQ) ${ }^{9}$ to reflect the reality of home isolation in the context of the COVID-19 pandemic. The Self-MOQ is a previously validated questionnaire that has good internal reliability (Cronbach $\alpha=0.84$ ) and validity $(r=-0.60, p<0.001) .{ }^{9}$ The questionnaire was unblinded, as patients were aware of their diagnostic test results, and was administered 3-15 days after sampling for RT-PCR. The interview included questions on clinical symptoms and comorbidities associated with anosmia and dysgeusia. The interviewers did not tell the interviewees that the primary purpose of the study was to assess the frequency of anosmia and dysgeusia. Patients who reported a decrease in smell or taste perception were asked more specific questions on those senses. All patients provided informed consent.

\section{Data analysis}

Data were transferred into an electronic input tool, Research Electronic Data Capture (REDCap, Vanderbilt University), and analyzed with Stata 15.1 (StataCorp). We compared continuous variables using the Mann-Whitney $U$ test. We selected variables to be included in the multivariable conditional logistic regression model according to the change-in-estimate criterion, in which confounders are defined as variables that alter the unadjusted exposure-outcome effect by a certain percentage. A cut-off of $10 \%$ was used. As anosmia and dysgeusia are closely related, they were considered together in multivariable models.

\section{Ethics approval}

This study was approved by the CHUS ethics review board.

\section{Results}

\section{Characteristics of cases and overall incidence}

Among 2883 people tested during the study period, we identified 146 who were positive for SARS-CoV-2. Of the 146,8 were excluded ( 3 had died, 4 were in the hospital at the time of the survey, and 1 declined to participate), and we were unable to find age-matched controls for 4 cases. We thus studied 134 patients who had tested positive for SARS-CoV-2 and 134 control patients. A total of 262 patients were already aware of the result of their test when the questionnaire was administered. Of the 134 patients who were positive for SARS-CoV-2, $70(52.2 \%)$ were women and 64 (47.8\%) were men; the median age was 57.1 (interquartile range 41.2-64.5) years. Only 3 SARSCoV-2-positive patients and 1 control patient had been admitted to hospital.

\section{Risk factors and symptoms of COVID-19}

Risk factors and symptoms associated with SARS-CoV-2 positivity in the bivariate analyses are presented in Table 1 . The odds ratio (OR) for the association of anosmia or dysgeusia or both with SARS-CoV-2 positivity was 20.0 (95\% confidence interval [CI] 7.3$54.6)$ and was not significantly different for women $(16.9,95 \% \mathrm{Cl}$ 7.6-37.4) and men (26.9, 95\% Cl 8.7-82.8). Several symptoms were associated with SARS-CoV-2 infection in the bivariate analysis but were no longer significant after adjustment for confounders. The independent symptoms associated with SARS-CoV-2 infection were anosmia or dysgeusia or both (adjusted OR 62.9, $95 \% \mathrm{Cl} 11.0-359.7$ ), presence of myalgia (adjusted OR 7.6, 95\% Cl 1.9-29.9), blurred vision (adjusted OR 0.1, 95\% $\mathrm{Cl} 0.0-0.8$ ) and chest pain (adjusted OR $0.1,95 \% \mathrm{Cl} 0.0-0.6$ ). The final multivariable regression model also included other variables that enhanced the fit of the model significantly but were not significantly associated with the outcome. These variables were loss of appetite (adjusted OR 2.2, 95\% Cl 0.8-5.9), sneezing (adjusted OR $0.4,95 \% \mathrm{Cl} 0.1-1.1$ ) and asthenia (adjusted OR $1.3,95 \% \mathrm{Cl}$ 0.5-3.3).

To further investigate the association between anosmia and dysgeusia and SARS-CoV-2 infection, we evaluated the gustatory and olfactory functions among SARS-CoV-2-positive patients who described a de novo olfactory or gustatory disorder (Table 2). Anosmia and dysgeusia were strongly correlated: 67 patients had both, 2 patients had only anosmia, and 18 patients had only dysgeusia $(p<0.001)$. Most patients described sudden and severe 
Table 1: Characteristics of patients who tested positive for severe acute respiratory syndrome coronavirus 2 and control patients

\section{No. $(\%)$ of patients*}

\section{Control patients \\ $n=134$}

$57.2(42.6-64.4)$

Age, yr, median (IQR)

Sex

Female

Male

Smoking

Never smoked

Past smoker

Active smoker

Seasonal allergies

Allergy to furry animals

Year-round nasal congestion

Year-round nasal drip

Long-term use of nasal corticosteroid sprays

No

Occasionally

Continuous use

Signs and symptoms (within $72 \mathrm{~h}$ before or after

SARS-CoV-2 testing)

Anosmia

Dysgeusia

Anosmia and/or dysgeusia

Asthenia

Myalgia

Arthralgia

Chest pain

Dyspnea

Chills

Fever (subjective)

Fever (objective)

Nasal congestion

Nasal drip

Sneezing

Sore throat

Cough

Sputum production

Loss of appetite

Nausea

Vomiting

Diarrhea

Headaches

Red eyes

Rash

Vertigo or dizziness

Blurred vision

Loss of temperature sensation in face
SARS-CoV-2-positive patients $n=134$

Crude OR $(95 \% \mathrm{CI})$

\author{
$57.1(41.2-64.5)$
}

$81(60.4)$

$53(39.6)$

$70(52.2)$

$64(47.8)$

1.0 (Ref.)

$1.3(0.8-2.1)$

$75(56.0)$

46 (34.3)

75 (56.0)

1.0 (Ref.)

$44(32.8)$

15 (11.2)

$16(11.9)$

20 (14.9)

17(12.7)

$14(10.4)$

16 (11.9)

$122(91.0)$

$6(4.5)$

$6(4.5)$

$12(9.0)$

18 (13.4)

15 (11.2)

124 (92.5)

$3(2.2)$

7 (5.2)

$0.9(0.5-1.7)$

$1.1(0.5-2.4)$

$0.8(0.4-1.6)$

$0.6(0.3-1.5)$

$1.3(0.6-2.7)$

$0.9(0.4-1.9)$

1.0 (Ref.)

$0.5(0.1-2 . .0)$

$1.2(0.4-3.5)$

$6(4.5)$

69 (51.5)

$32.5(8.0-132.7)$

$9(6.7)$

85 (63.4)

$16.2(6.6-40.0)$

$11(8.2)$

$87(64.9)$

$20.0(7.3-54.6)$

$58(43.3)$

$104(77.6)$

$3.9(2.2-6.7)$

$76(56.7)$

$4.9(2.6-9.2)$

19 (14.2)

$37(27.6)$

$2.2(1.2-4.1)$

35 (26.1)

$1.2(0.7-2.2)$

49 (36.6)

$56(41.8)$

$1.2(0.8-2.0)$

71 (53.0)

$46(34.3)$

$50(37.3)$

$58(43.3)$

$60(44.8)$

$53(39.6)$

$60(44.8)$

97 (72.4)

$40(29.8)$

$75(56.0)$

$40(29.8)$

$9(6.7)$

60 (44.8)

87 (64.9)

$1(0.7)$

$8(6.0)$

$27(20.1)$

6 (4.5)

$5(3.7)$
$2.7(1.7-4.7)$

$1.5(0.9-2.6)$

$2.9(1.6-5.1)$

$1.1(0.6-1.9)$

$0.6(0.3-1.0)$

$0.8(0.5-1.4)$

$0.7(0.4-1.1)$

$1.0(0.6-1.7)$

$0.9(0.6-1.5)$

$4.1(2.4-7.0)$

$2.9(1.5-5.6)$

$1.8(0.6-5.4)$

$2.7(1.6-4.7)$

$2.9(1.3-3.4)$

$0.3(0.0-3.2)$

$1.4(0.4-4.4)$

$2.3(1.1-4.8)$

$0.7(0.2-1.9)$

$5.0(0.6-42.9)$

Note: $\mathrm{Cl}=$ confidence interval, $\mathrm{IQR}=$ interquartile range, $\mathrm{OR}=$ odds ratio, Ref. = reference, $\mathrm{SARS}-\mathrm{CoV}-2$ = severe acute respiratory syndrome coronavirus 2.

${ }^{\star}$ Except where noted otherwise. 
Table 2: Characteristics of patients who tested positive for severe acute respiratory syndrome coronavirus 2 and had anosmia or dysgeusia

\begin{tabular}{|c|c|}
\hline Characteristic & patients* \\
\hline \multicolumn{2}{|l|}{$\begin{array}{l}\text { Self-described change in olfactory capacity } \\
(n=69)\end{array}$} \\
\hline Light & $6(9)$ \\
\hline Moderate & $12(17)$ \\
\hline Severe & $51(74)$ \\
\hline \multicolumn{2}{|l|}{ Onset of change in olfactory capacity $(n=69)$} \\
\hline Progressive (over several days) & $19(28)$ \\
\hline Sudden & $50(72)$ \\
\hline \multicolumn{2}{|l|}{ Capacity to recognize specific odours $(n=69)$} \\
\hline \multicolumn{2}{|l|}{ Perfume } \\
\hline Not at all & $38(55)$ \\
\hline Weak & $22(34)$ \\
\hline Good & $3(4)$ \\
\hline Very good & $1(2)$ \\
\hline Not applicable & $5(7)$ \\
\hline \multicolumn{2}{|l|}{ Smoke } \\
\hline Not at all & $32(46)$ \\
\hline Weak & $17(25)$ \\
\hline Good & $5(7)$ \\
\hline Very good & $1(2)$ \\
\hline Not applicable & $14(20)$ \\
\hline \multicolumn{2}{|l|}{ Garbage } \\
\hline Not at all & $36(52)$ \\
\hline Weak & $21(30)$ \\
\hline Good & $2(3)$ \\
\hline Very good & $1(2)$ \\
\hline Not applicable & $9(13)$ \\
\hline \multicolumn{2}{|l|}{ Coffee } \\
\hline Not at all & $35(51)$ \\
\hline Weak & $27(39)$ \\
\hline Good & $2(3)$ \\
\hline Very good & $1(2)$ \\
\hline Not applicable & $4(6)$ \\
\hline $\begin{array}{l}\text { Modification/alteration in tasting capacity } \\
(n=85)\end{array}$ & $31(37)$ \\
\hline Loss of tasting capacity $(n=85)$ & $68(81)$ \\
\hline \multicolumn{2}{|l|}{ Onset of change in tasting capacity $(n=85)$} \\
\hline Progressive (over several days) & $33(39)$ \\
\hline Sudden & $52(61)$ \\
\hline \multicolumn{2}{|l|}{$\begin{array}{l}\text { Self-described change/alteration in tasting } \\
\text { capacity }(n=85)\end{array}$} \\
\hline Light & $8(9)$ \\
\hline Moderate & $31(36)$ \\
\hline Severe & $46(54)$ \\
\hline
\end{tabular}

loss in their olfactory or gustatory abilities, or both. This loss was reflected in their ability to recognize perfume, smoke, garbage and coffee odours.

The symptoms most commonly described as presenting symptoms by SARS-CoV-2-positive patients were sore throat (21 patients [15.7\%]) and cough (39 [29.1\%]). Anosmia and dysgeusia were not reported frequently as presenting manifestations of SARS-CoV-2 positivity ( 3 patients [2.2\%] and 2 patients [1.5\%], respectively). The most severe symptoms reported by respondents were cough (26 patients [19.4\%]), asthenia (22 [16.4\%]) and headache (16 [11.9\%]). Anosmia (4 patients [3.0\%]) and dysgeusia (5 [3.7\%]) were reported infrequently as the most severe symptoms experienced. Anosmia or dysgeusia, or both, without fever or cough, were observed in almost one-third of patients (16 of 57 [28\%]) but were reported as the sole symptom in only 2 of 134 patients (1.5\%).

\section{Interpretation}

We found that anosmia and dysgeusia were the most distinctive symptoms associated with SARS-CoV-2 infection and that these symptoms could be severe, as shown by the complete loss of perception of odours such as coffee and garbage. Because the study took place during a period when the criteria for SARSCoV-2 testing included symptomatic (fever, cough or dyspnea) travellers and contacts of confirmed COVID-19 cases, it underlines the signs and symptoms that may be characteristic of SARSCoV-2 infection compared to infection with other respiratory viruses that were prevalent in our community in March 2020.

Other studies have suggested anosmia to be an important symptom of COVID-19. Olfactory and taste disorders were described in reports ${ }^{10,11}$ and in descriptive studies of patients with mild to moderate COVID-19 ${ }^{12}$ and those admitted to hospital. ${ }^{6}$ These symptoms appeared to be less prominent in cohorts of inpatients: Giacomeli and colleagues ${ }^{6}$ reported that $39(66 \%)$ of 59 patients admitted to hospital did not report any taste or olfactory disorders. Inpatients may have other, more severe symptoms to focus on, do not have access to familiar odours such as garbage, perfume or coffee, and have other health concerns that may mitigate the perception of olfactory disorders.

Our findings are similar to those of a study of 417 patients with mild to moderate COVID-19 in which olfactory and gustatory dysfunction was reported in $85.6 \%$ and $88.0 \%$ of patients, respectively. ${ }^{12}$ They are in keeping with results from a crosssectional study conducted in California in which anosmia (adjusted OR 10.9) and ageusia (adjusted OR 10.2) were associated with COVID-19 positivity. ${ }^{13}$ Our results also align with those of recent Italian and German studies showing altered sense of smell or taste in $64.4 \%$ and $69 \%$ of patients, respectively. ${ }^{7,14}$

About $5 \%$ of the general population exhibit functional anosmia. ${ }^{15}$ Without comparison to matched controls, determining the magnitude of the potential association between anosmia or dysgeusia, or both, and COVID-19 is difficult. Our study reinforces the association between SARS-Cov-2 positivity and olfactory or gustatory dysfunction. Our control patients consisted primarily 
of people with influenza-like illness who had potentially been exposed to SARS-CoV-2, predominantly through travel. This feature allowed us to differentiate anosmia related to SARS-CoV-2 from nonspecific anosmia associated with other respiratory viruses or with nasal congestion. ${ }^{15-17}$ The anosmia described by our SARS-CoV-2-positive patients was independent of nasal congestion. Patients who experienced sneezing had lower odds of infection. Beltrán-Corbellini and colleagues ${ }^{18}$ used influenzapositive historical controls to determine the relation between acute-onset smell and taste disorders in patients with SARSCoV-2 infection and reported similar findings to ours: they found that new-onset smell or taste disorders were significantly more frequent among patients who had tested positive for SARS-CoV-2 $(39.2 \%)$ than in the control group (12.5\%).

Severe acute respiratory syndrome coronavirus 2 may cause neurologic manifestations, ${ }^{19}$ and other coronaviruses have been linked to nervous system involvement and neuron-to-neuron propagation via axonal transport. ${ }^{20}$ Severe acute respiratory syndrome coronavirus 2 has been linked to olfactory neuropathy. ${ }^{21} \mathrm{~A}$ study in transgenic mice showed that the virus can infect the olfactory bulb neurons and reach the central nervous system via transsynaptic spread. ${ }^{22}$ These examples underline the biologic plausibility of olfactory disorders in SARS-CoV-2-positive patients.

\section{Limitations}

The main limitation of our study is its retrospective nature, which may have led to recall bias. However, blinding interviewers to participants' disease status was not possible. Most patients were aware of their test result, and it is likely that some of them had become aware of the association between anosmia and COVID19 at the time of the study, as these symptoms were emphasized publicly when a National Basketball Association player who had tested positive for SARS-CoV-2 reported them via Twitter. ${ }^{23}$ Around the time of our survey, media reports were already suggesting de novo anosmia as a potential symptom of COVID-19. Therefore, the SARS-CoV-2-positive patients who were aware of their test results may have been more prone to report olfactory and gustatory disorders. Another potential bias relates to misclassification of cases. Reverse transcription polymerase chain reaction testing for SARS-CoV-2 is not $100 \%$ sensitive, and positivity can depend on factors such as the number of days from onset of symptoms and the site of sampling. ${ }^{24,25}$ However, this differential misclassification of outcome is likely to underestimate the true association between olfactory and gustatory dysfunction and SARS-CoV-2 positivity, and we found a strong association.

\section{Conclusion}

In this age-matched case-control study, we found a strong association between olfactory and gustatory symptoms and SARSCoV-2 positivity. These symptoms affect the ability to recognize odours frequently encountered in daily life and are independent from nasal congestion and nasal drip, which were observed more frequently in our control population. These results reinforce the suggestion that anosmia and dysgeusia should be considered common and distinctive features of SARS-CoV-2 infection and indications for testing. Given the recent concerns about the imperfect sensitivity of RT-PCR testing, the association between anosmia or dysgeusia with SARS-CoV-2 is strong enough that the presence of these symptoms should serve as an indication for retesting in patients whose initial test result is negative. Prospective studies should assess whether these symptoms can be permanent.

\section{References}

1. Hopkins C, Kumar N. Loss of sense of smell as marker of COVID-19 infection. London: ENT UK at The Royal College of Surgeons of England; 2020. Available: https://www.entuk.org/sites/default/files/files/Loss\%20of\%20sense\%20of\%20 smell\%20as\%20marker\%20of\%20COVID.pdf (accessed 2020 Apr. 16).

2. Huang C, Wang Y, Li X, et al. Clinical features of patients infected with 2019 novel coronavirus in Wuhan, China. Lancet 2020;395:497-506.

3. Guan WJ, Ni ZY, Hu Y, et al. Clinical characteristics of coronavirus disease 2019 in China. N Engl J Med 2020;382:1708-20.

4. Wang D, Hu B, Hu C, et al. Clinical characteristics of 138 hospitalized patients with 2019 novel coronavirus-infected pneumonia in Wuhan, China. JAMA 2020; 323:1061-9.

5. Wu Z, McGoogan JM. Characteristics of and important lessons from the coronavirus disease 2019 (COVID-19) outbreak in China: summary of a report of 72314 cases from the Chinese Center for Disease Control and Prevention. JAMA 2020 Feb. 24 [Epub ahead of print]. doi: 10.1001/jama.2020.2648.

6. Giacomelli A, Pezzati L, Conti F, et al. Self-reported olfactory and taste disorders in SARS-CoV-2 patients: a cross-sectional study. Clin Infect Dis 2020 Mar. 26. pii: ciaa330 [Epub ahead of print]. doi: 10.1093/cid/ciaa330.

7. Spinato G, Fabbris C, Polesel J, et al. Alterations in smell or taste in mildly symptomatic outpatients with SARS-CoV-2 infection. JAMA 2020 Apr. 22 [Epub ahead of print]. doi: 10.1001/jama.2020.6771.

8. Konstantinidis I, Haehner A, Frasnelli J, et al. Post-infectious olfactory dysfunction exhibits a seasonal pattern. Rhinology 2006;44:135-9.

9. Zou LQ, Linden L, Cuevas M, et al. Self-reported Mini Olfactory Questionnaire (Self$\mathrm{MOQ}$ ): a simple and useful measurement for the screening of olfactory dysfunction. Laryngoscope 2019 Nov. 20 [Epub ahead of print]. doi: 10.1002/lary.28419.

10. Gane SB, Kelly C, Hopkins C. Isolated sudden onset anosmia in COVID-19 infection. A novel syndrome? Rhinology 2020 Apr. 2 [Epub ahead of print]. doi: 10.4193/Rhin20.114.

11. Eliezer M, Hautefort C, Hamel AL, et al. Sudden and complete olfactory loss function as a possible symptom of COVID-19. JAMA Otolaryngol Head Neck Surg 2020 Apr. 8 [Epub ahead of print]. doi: 10.1001/jamaoto.2020.0832.

12. Lechien JR, Chiesa-Estomba CM, De Siati DR, et al. Olfactory and gustatory dysfunctions as a clinical presentation of mild-to-moderate forms of the coronavirus disease (COVID-19): a multicenter European study. Eur Arch Otorhinolaryngol 2020 Apr. 6 [Epub ahead of print]. doi: 10.1007/s00405-020-05965-1.

13. Yan CH, Faraji F, Prajapati DP, et al. Association of chemosensory dysfunction and COVID-19 in patients presenting with influenza-like symptoms. Int Forum Allergy Rhino 2020 Apr. 20 [Epub ahead of print]. doi: 10.1002/alr. 22579.

14. Luers JC, Rokohl AC, Loreck N, et al. Olfactory and gustatory dysfunction in Coronavirus disease 19 (COVID-19). Clin Infect Disease 2020 May 1. doi: 10.1093/ cid/ciaa525

15. Hummel T. Perspectives in olfactory loss following viral infections of the upper respiratory tract. Arch Otolaryngol Head Neck Surg 2000;126:802-3.

16. Fornazieri MA, Borges BB, Bezerra TF, et al. Main causes and diagnostic evaluation in patients with primary complaint of olfactory disturbances. [article in English, Portuguese]. Braz J Otorhinolaryngol 2014;80:202-7.

17. Suzuki M, Saito K, Min WP, et al. Identification of viruses in patients with postviral olfactory dysfunction. Laryngoscope 2007;117:272-7.

18. Beltrán-Corbellini Á, Chico-García JL, Martínez-Poles J, et al. Acute-onset smell and taste disorders in the context of COVID-19: a pilot multicenter PCRbased case-control study. Eur J Neurol Apr. 22 [Epub ahead of print]. doi: 10. 1111/ene.14273.

19. Moriguchi T, Harii N, Goto J, et al. A first case of meningitis/encephalitis associated with SARS-Coronavirus-2. Int J Infect Dis 2020;94:55-8.

20. Dubé $M$, Le Coupanec $A$, Wong AHM, et al. Axonal transport enables neuron-toneuron propagation of human coronavirus OC43. J Virol 2018;92:e00404-18. 
21. Hwang CS. Olfactory neuropathy in severe acute respiratory syndrome: report of a case. Acta Neurol Taiwan 2006;15:26-8.

22. Netland J, Meyerholz DK, Moore S, et al. Severe acute respiratory syndrome coronavirus infection causes neuronal death in the absence of encephalitis in mice transgenic for human ACE2. J Virol 2008;82:7264-75.

23. Gobert R. Just to give you guys an update, loss of smell and taste is definitely one of the symptoms, haven't been able to smell anything for the last 4 days. Anyone experiencing the same thing? [Tweet]. 2020 Mar. 22.
24. Lippi G, Simundic AM, Plebani M. Potential preanalytical and analytical vulnerabilities in the laboratory diagnosis of coronavirus disease 2019 (COVID19). Clin Chem Lab Med 2020 Mar. 16 [Epub ahead of print]. pii: /j/cclm. ahead-of-print/cclm-2020-0285/cclm-2020-0285.xml. doi: 10.1515 / cclm-2020-0285.

25. Wang W, Xu Y, Gao R, et al. Detection of SARS-CoV-2 in different types of clinical specimens. JAMA 2020; Mar. 11 [Epub ahead of print]. doi: 10.1001/jama.2020.3786.

\section{Competing interests: None declared.}

This article has been peer reviewed.

Affiliations: Departments of Microbiology and Infectious Diseases (Carignan, L. Valiquette, Grenier, Musonera, Nkengurutse, Marcil-Héguy, Vettese, Marcoux, C. Valiquette, Xiong, Pépin), Surgery (Fortier) and Community Health Sciences (Généreux), Université de Sherbrooke, Sherbrooke, Que.

Contributors: Alex Carignan, Cynthia Grenier, Dominique Marcoux and Jacques Pépin supervised the project. Alex Carignan, Louis Valiquette, Cynthia Grenier, Pierre-Hughes Fortier and Jacques Pépin conceived the study. Alex Carignan, Louis Valiquette, Cynthia
Grenier, Jean Berchmans Musonera, Delphin Nkengurutse, Anaïs Marcil-Héguy, Kim Vettese, Corinne Valiquette and Jacques Pépin designed the study. Cynthia Grenier, Jean Berchmans Musonera, Delphin Nkengurutse, Anaïs Marcil-Héguy, Kim Vettese, Dominique Marcoux, Corinne Valiquette and Wei Ting Xiong acquired the data. Alex Carignan, Louis Valiquette and Jacques Pépin analyzed and interpreted the data. Alex Carignan, Louis Valiquette, Wei Ting Xiong and Jacques Pépin drafted the manuscript, and Alex Carignan, Louis Valiquette, Cynthia Grenier, Jean Berchmans Musonera, Delphin Nkengurutse, Anaïs Marcil-Héguy, Kim Vettese, Corinne Valiquette, Wei Ting Xiong, Pierre-Hughes Fortier, Mélissa Généreux and Jacques Pépin revised it critically for important intellectual content. All of the authors approved the final version to be submitted and agreed to be accountable for all aspects of the work.

Funding: This work was supported by the Centre de recherche du Centre hospitalier universitaire de Sherbrooke.

Data sharing: All or portions of the data are available to others; data will be available on publication; and data may be accessed by contacting the corresponding author.

Accepted: May. 14, 2020

Correspondence to: Alex Carignan, Alex. Carignan@USherbrooke.ca 